\title{
The study of using precise measurement to evaluate the outcomes after ear reconstruction
}

\author{
Peng Zhu and Sui-jun Chen* \\ Department of Otolaryngology, Sun Yat-sen Memorial Hospital, Sun Yat-sen University, Guang Zhou, China
}

\begin{abstract}
Objective: Microtia ear reconstruction is one of the most challenging operations in plastic surgery, the aim of the present study is to use a new method to evaluate the results after ear reconstruction.

Study design: Observational study.

Setting: University hospital from December 2016 to January 2018.

Subjects and methods: In total, 40 children underwent autologous ear reconstruction with three-stage technique, 20 patients who had a 3D digital template made for clinical application, and the other 20 who had undergone ear reconstruction with a $2 \mathrm{D}$ template, all of the subjects had been on a more than 3 -months postoperative follow-up. For the auricle's 20 precise measurements which count, combined with satisfaction questionnaires, a complete review of anatomic features, surgical results were performed.
\end{abstract}

Results: The measurement results of the 3D model group remanufactured ear profile were more correlated with the measurement results of the healthy side ear profile, while the patients in the 3D model group were more satisfied with the reconstructed ear profile, which was consistent with the clinical actual results, and objectively reflected the effect of the patient after the operation.

Conclusion: The application of 3D model is helpful to improve the effect of surgery, and the precise measurement evaluation method combined with the satisfaction questionnaire can reflect the patient's postoperative effect in a realistic and objective way.

\section{Introduction}

Being born with microtia, which is a rare congenital ear deformity, can be a severe handicap with complex effects on the parent's life. Microtia ear reconstruction is one of the most challenging operations in plastic surgery [1]. Its core is the ear framework and the covering skin. The current mainstream method uses autologous rib cartilage to complete the ear reconstruction [2-4]. The human ear is difficult to reproduce surgically because it is composed of a complex, convoluted frame of delicate elastic cartilage surrounded by a thin skin envelope. Regardless of the size and shape of the deformity, the ultimate goal of total reconstruction of the ear is the construction of an auricle with an appearance as close as possible to that of the normal ear. The evaluation of microtia after ear reconstruction should consider their clinical behaviour and biological characteristics.

\section{Materials and methods}

This study was performed in the Department of Otolaryngology at the Sun Yat-sen Memorial Hospital of Sun Yat-sen University. Forty patients with unilateral congenital microtia, who had undergone autologous rib cartilage ear reconstruction with three-stage technique [5] were enrolled in this study, they were divided into two groups randomly before surgery, 20 patients who had a $3 \mathrm{D}$ digital template [6-8] made for clinical application (Figure 1), and the other 20 who had undergone ear reconstruction with a 2D template (Figure 2). All these surgeries were performed by Sui-jun Chen, who has more than 5 years' experience of ear reconstruction surgery. The Nagata technique for auricular reconstruction has been used in all patients [9].
All the subjects had been on a more than 3-months postoperative follow-up. In the 3D model group, there is a strong resemblance in the location and appearance between the reconstructed ear and the healthy ear (Figure 3), while the location and appearance between the reconstructed ear and the healthy ear after ear reconstruction with a $2 \mathrm{D}$ template model are not as good as the $3 \mathrm{D}$ ones (Figure 4).

For the auricle's 20 precise measurements which count, the result was given based on the 20 precise measurements of the reconstructed auricle with the contralateral normal auricle. First, we locate the 17 coordinate points of the ear profile, and then measure a total of 20 sets of data for each subatomic structure unit of the reconstructed ear profile and the side health ear profile respectively, respectively, calculate the correlation coefficient of the reconstructed ear and healthy side ear profile measurement data, compare and analyse the results obtained by the $3 \mathrm{D}$ model group and the $2 \mathrm{D}$ plane model group.

A questionnaire, as an instrument to measure patient's satisfaction, was created to get a multidimensional view. Statements were created within the following four domains: appearance of my new ear, shape

${ }^{\star}$ Correspondence to: Sui-jun Chen, Department of Otolaryngology, Sun Yatsen Memorial Hospital, Sun Yat-sen University, Guang Zhou, China, Tel: +86 208133 2199; E-mail: chensuij@mail.sysu.edu.cn

Key words: microtia ear reconstruction, $3 D$ model precise measurement

Received: August 12, 2019; Accepted: August 26, 2019; Published: August 29, 2019 


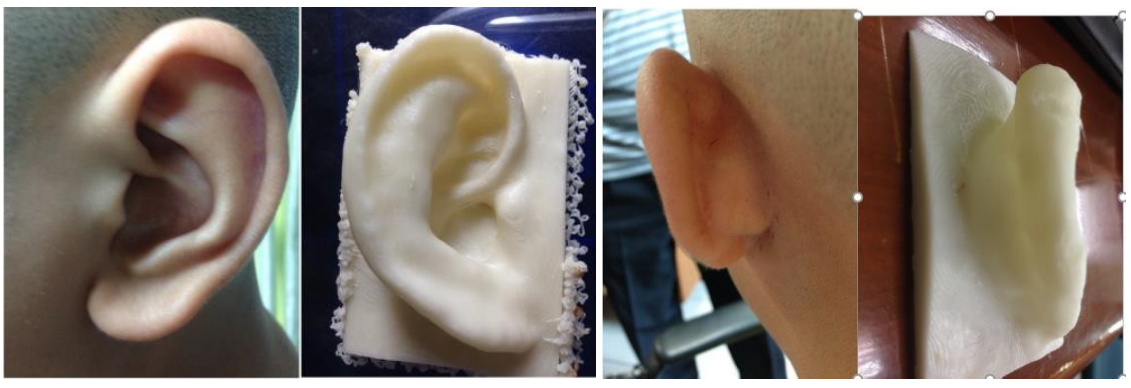

Figure 1. A 3D digital template made for clinical application

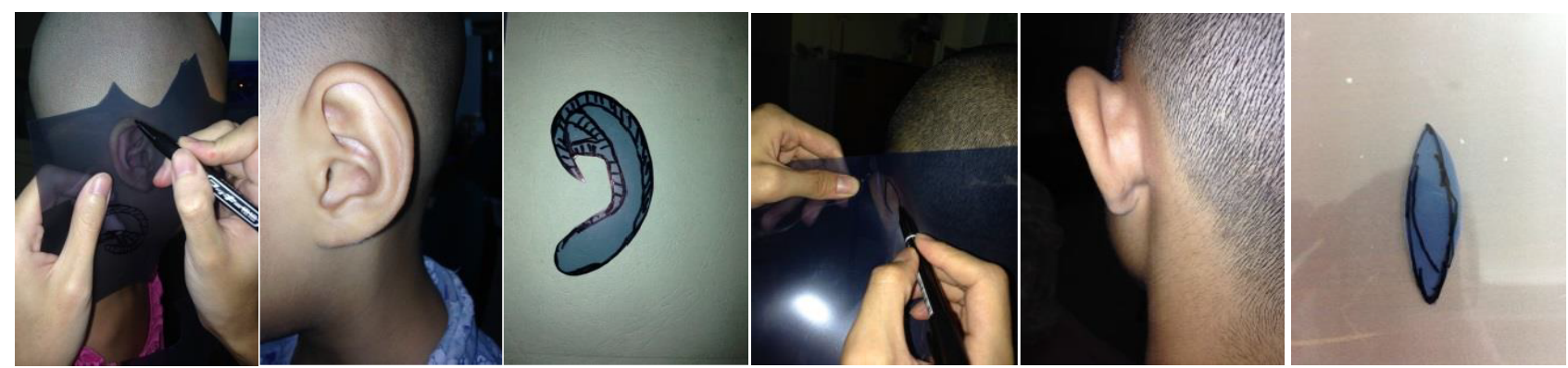

Figure 2. 2D template model before surgery.

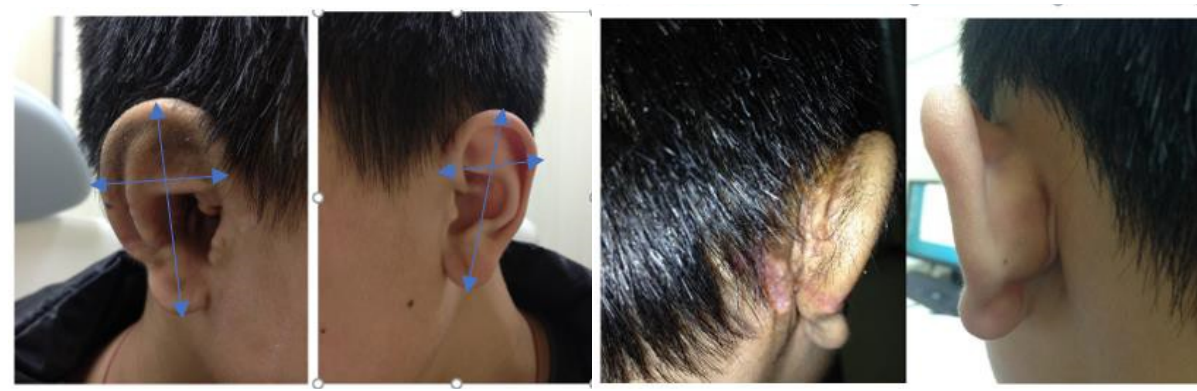

Figure 3. Clinical outcomes of the 3D model group and precise.

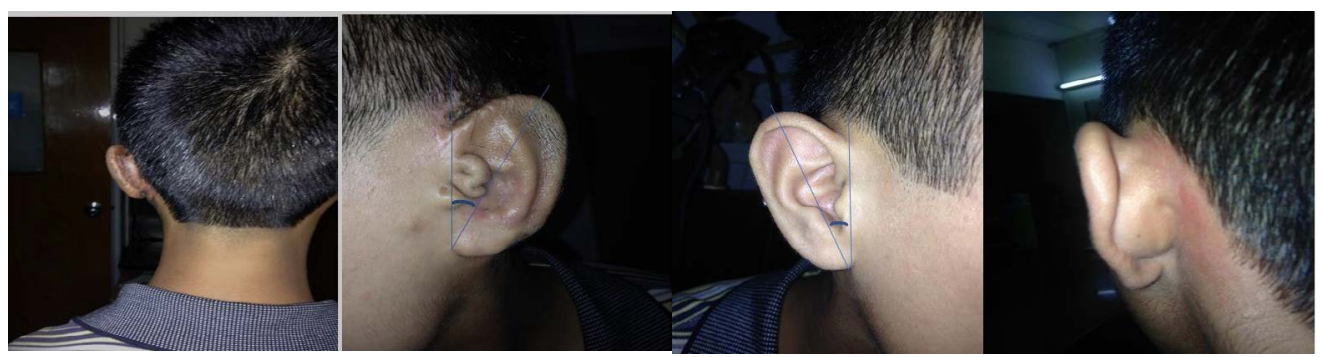

Figure 4. Clinical outcomes of the 2D model group and precise.

of my new ear, similar to the other one, and size of my new ear [10]. Fully disagree score $=0$ points, disagree score $=3$ points, agree score $=$ 7 points, fully agree score $=10$ points, analyse the results obtained by the $3 \mathrm{D}$ model group and the $2 \mathrm{D}$ plane model group.

The landmarks of the auricle are the: (1) Superaurale, (2) Subaurale, (3) Preaurale, (4) Postaurale, (5) Superior crura anthelicis, (6) Inferior crura anthelicis, (7) Incisura anterior Auris Posterior, (8) Strongest Antihelical Curvature, (9) Midportion of Helical Rim, (10) Cavum Conchae (Deepest Portion), (11) Antitragus, (12) Lobule Posterior, (13) Lobule Anterior, (14) Incisura IntertragicaInferior, (15) Tragus, (16) superior Auriculocephalic angle, and (17) inferior Auriculocephalic angle (Figure 5).
A total of 20 anthropometric measurements were taken [11].

- Auricular length (1-2). Distance from the most superior pole of the helical rim (superaurale) to the most inferior pole of the lobe (subaurale).

- Auricular width (3-4). Distance from the preaurale to the postaurale.

- Conchal length (5-14). Distance from concha superior to incisura intertragica inferior.

- Crura anthelicis length (5-6). Distance from Superior crura anthelicis to Inferior crura anthelicis.

- Conchal width (7-8). Distance from incisura anterior auris to strongest antihelical curvature. 


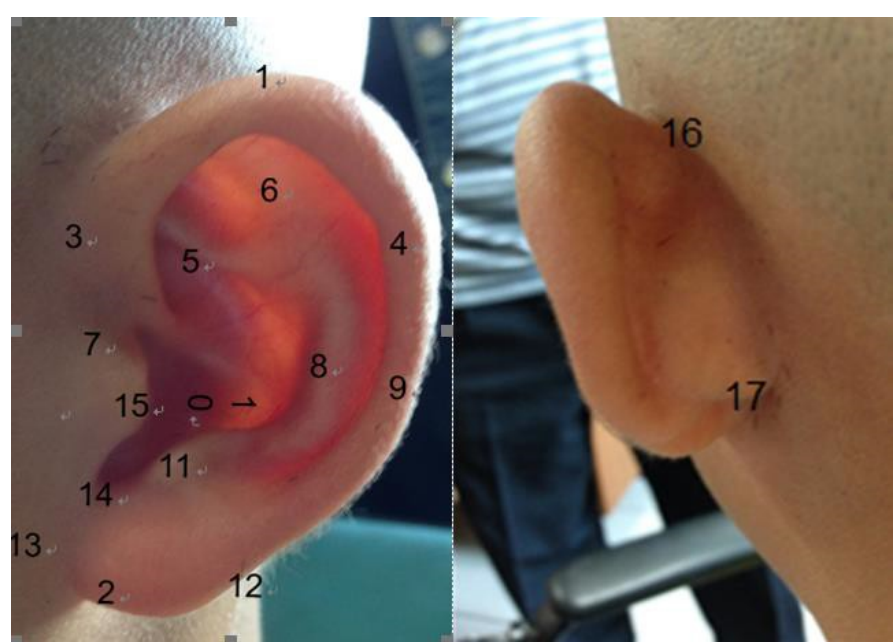

Figure 5. Seventeen landmarks of the auricle.

- Antihelical angle (6-11). Angle from Inferior crura anthelicis to Antitragus.

- Antihelical helight (6-11). Distance from Inferior crura anthelicis to Antitragus.

- the conchal bowl.

- Depth of conchal (10-7/8) From the bottom of the conchal to the midpoint of the 7-8 connection

- Lobule length (14-2). Distance from incisura intertragica inferior to subaurale.

- Lobule width (12-13). Distance from lobule posterior to lobule anterior.

- Intertragal distance (11-15). Distance from tragus to antitragus.

- Lobule posterior to antitragus (11-12). Antihelix to helix at the strongest point.

- Traguslength (7-14). Distance from Incisura anterior Auris Posterior to Incisura.

- Auriculocephalic angle (16-17). The angle from superior Auriculocephalic angle to inferior Auriculocephalic angle

- Auriculocephalic angle height (16-17). The distance from superior Auriculocephalic angle to inferior Auriculocephalic angle.

- Upper third projection at superaurale level to mastoid.

- Middle third projection at mid helix to mastoid.

- Lower third projection at lobule posterior level to Mastoid.

- The axis fo the ear (3/13-1/2) Angle between points 13 and 3 and points 1 and 2

- Distance between the Antihelical and the helical in the most bending place (8-9)

\section{Statistics}

SPSS version 17.0 was used in all data analyses. Paired T-test and Spearman analysis were used to determine the difference between the two different adjuvant template models. A difference with a p-value < 0.05 was considered as statistically significant.

\section{Ethical considerations}

This study was approved by the ethics committee of our institute. Informed consent for surgery was obtained from all of the children's parents.

\section{Results}

Comparing the data among the two groups, there are significant differences for each measurement. The $3 \mathrm{D}$ model group's correlation factor (Table 1) and patient satisfaction scores were statistically higher that of the 2D model group (Figures 6 and 7).

\section{Discussion}

Microtia is a well-known craniofacial malformation of the auricle, comprising a clinical spectrum ranging from a slight reduction in the size of the auricle to the complete absence of the auricle [12-14]. The reconstruction of an auricle for congenital deformity or following trauma remains one of the greatest challenges in reconstructive surgery. The traditional 2D film in X-rays has difficulties in meeting the needs of sophisticated plastic surgery. The expanded use of $3 \mathrm{D}$ printers in the field of medicine has led to a number of innovations, especially through the building of patient-specific models based on actual imaging data, we have been able to create models that are accurate both anatomically and spatially. These models duplicate actual tissue and, thus, provide an element of realism that may be helpful for surgeons to get a better result after ear reconstruction. Moreover, these subtle structures are basically incorporated into the postoperative evaluation method sought through accurate measurement, making it more compliant.

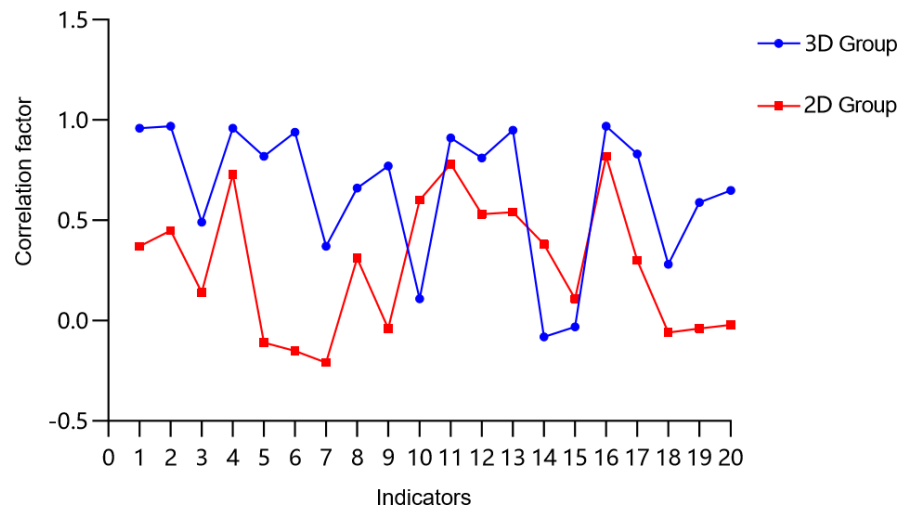

Figure 6. Correlation factor of 3D group is higher than that of 2D group

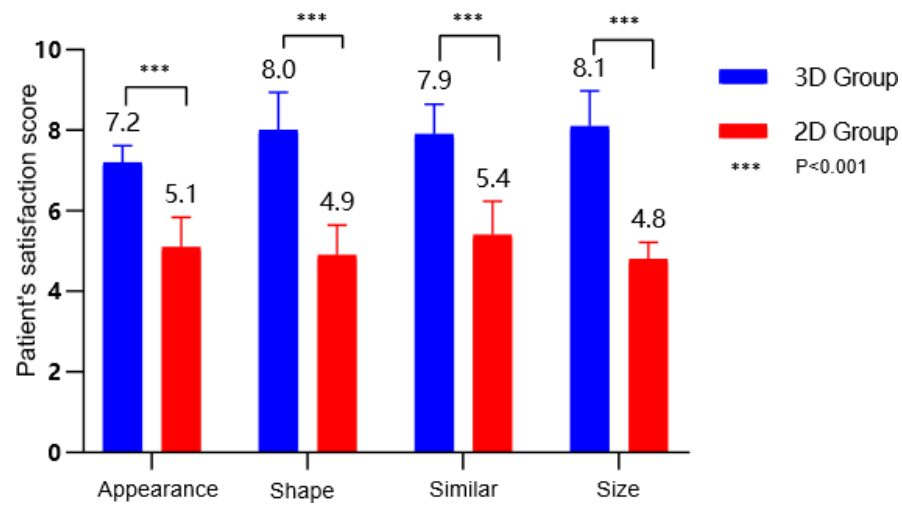

Figure 7. Patient's satisfaction of $3 \mathrm{D}$ group is higher than that of $2 \mathrm{D}$ group 
Table 1. The results of correlation factor between the reconstructed ear and the healthy ear

\begin{tabular}{|c|c|c|c|c|}
\hline & $3 \mathrm{D}$ group & $3 \mathrm{D}$ group & $2 \mathrm{D}$ group & $2 \mathrm{D}$ group \\
\hline & $\begin{array}{l}\text { Spearman Correlation } \\
\text { factor }\end{array}$ & P Value & $\begin{array}{l}\text { Spearman Correlation } \\
\text { factor }\end{array}$ & P Value \\
\hline Auricular length & 0.96 & $<.0001$ & 0.37 & 0.29 \\
\hline Auricular width & 0.97 & $<.0001$ & 0.45 & 0.19 \\
\hline Conchal length & 0.49 & 0.15 & 0.14 & 0.70 \\
\hline Conchal width & 0.96 & $<.0001$ & 0.73 & 0.02 \\
\hline Depth of conchal & 0.82 & $<.0001$ & -0.11 & 0.77 \\
\hline Lobule length & 0.94 & $<.0001$ & -0.15 & 0.69 \\
\hline Lobule width & 0.37 & 0.29 & -0.21 & 0.56 \\
\hline Intertragal distance & 0.66 & 0.04 & 0.31 & 0.39 \\
\hline Lobule posterior to antitragus & 0.77 & 0.01 & -0.04 & 0.91 \\
\hline Distance between the antihelical and the helica & 0.11 & 0.75 & 0.60 & 0.07 \\
\hline Upper third projection at superaurale level to mastoid & 0.91 & $<.0001$ & 0.78 & 0.01 \\
\hline Middle third projection at mid helix to mastoid & 0.81 & $<.0001$ & 0.53 & 0.11 \\
\hline Lower third projection at lobule posterior level to mastoid & 0.95 & $<.0001$ & 0.54 & 0.11 \\
\hline Auriculocephalic angle & -0.08 & 0.84 & 0.38 & 0.28 \\
\hline Auriculocephalic angle height & -0.03 & 0.94 & 0.11 & 0.77 \\
\hline Traguslength & 0.97 & $<.0001$ & 0.82 & 0.00 \\
\hline Anthelicis length & 0.83 & $<.0001$ & 0.30 & 0.40 \\
\hline The axis of the ear & 0.28 & 0.44 & -0.06 & 0.86 \\
\hline Antihelical angle & 0.59 & 0.08 & -0.04 & 0.92 \\
\hline Antihelical helight & 0.65 & 0.04 & -0.02 & 0.96 \\
\hline
\end{tabular}

At present, there is no unified and objective method for evaluating the effect of ear reconstruction, Nakai has put forward the standard of ear reconstruction [15], which gives us the direction of efforts as a whole, but there is still no quantifiable method to evaluate the effect of surgery, Without violating Nakai's standards, combined with our clinical work, we have proposed a quantifiable method for evaluating the effect of surgery by means of precise measurement, and the results show that the precise measurement results of the reconstructed ear of patients in the 3D model group are similar to those of the healthy ear [8]. Patients' satisfaction with reconstructed ear in the 3D model group is better than that of the $2 \mathrm{D}$ model group, consistent with the clinical actual results, the method of precise measurement is more objective and accurate, and we can expect to use artificial intelligence (AI) [1618] technology to automatically obtain the precise measurement results, further improve the accuracy of the evaluation, objectivity, evaluation process is more simple.

China began to pay attention to precision medicine as early as the beginning of this century, and in 2006 for the first time put forward the concept of precision medicine, has been recognized by the domestic and foreign medical communities, and applied to the field of cancer radiotherapy, maternity and other medical fields, precision medicine compared to traditional empirical medicine has made great progress, science through precision instruments, Advanced modern technologies such as life sciences are integrated with traditional experience to greatly reduce the uncertainty of clinical practice, thus achieving in surgery "the cut of the piece not left, the remaining unscathed", in order to ensure precision and as far as possible to minimize damage control. With the continuous improvement of patient's cognitive level and the maturity and development of surgical reconstruction technology, the whole ear reconstruction has entered a new stage of development, and also put forward higher requirements for the surgeon, and the shape of the ear is particularly complex, three-dimensional sense strong, want to recreate a realistic shape and similar to the opposite side of the ear is not easy, relative to the traditional 2D model, 3D printing auxiliary ear model can more realistic, accurate reproduction of the various subatomic structure units and orientation of the healthy ear, through the precise measurement of reconstructed ear and the healthy ear, proposed an objective precise method for evaluating the patient's postoperative effect.

\section{Conclusion}

With the development of surgical technology, it is not impossible to recreate a forced ear. And we apply precise measurement of postoperative evaluation method basically covers the various subatomic structure units of the ear, more in line with the requirements of modern surgery.

\section{References}

1. Cho BC, Kim JY, Jin SB (2007) Two-stage reconstruction of the auricle in congenital microtia using autogenous costal cartilage. J Plast Reconstr Aesthet Surg 60: 998-1006. [Crossref]

2. Dellacroce FJ, Green S, Rd AE (2001) Framework growth after reconstruction for microtia: is it real and what are the implications? Plast Reconstr Surg 108: 1479. [Crossref]

3. Kelley PE, Scholes MA (2007) Microtia and Congenital Aural Atresia. Otolaryng Clin $N A m$ 40: 61-80. [Crossref]

4. Vanek J, Galicia JAG, Benes B, Mech R, Carr N, et al. (2015) PackMerger: A 3D Print Volume Optimizer. Comput Graph Forum 33: 322-332.

5. Firmin F (1998) Ear reconstruction in cases of typical microtia. Personal experience based on 352 microtic ear corrections. Scand J Plast Reconstr Surg Hand Surg 32: 35-47. [Crossref]

6. Ito K, Gomi Y, Sato S, Arai Y, Shinoda K (2010) Clinical application of a new compact CT system to assess 3-D images for the preoperative treatment planning of implants in the posterior mandible A case report. Clin Oral Implants Res 12: 539-542. [Crossref]

7. Ventola CL (2014) Medical Applications for 3D Printing: Current and Projected Uses. P T 39: 704-711. [Crossref]

8. Stopp S, Wolff T, Irlinger F, Lueth T (2008) A new method for printer calibration and contour accuracy manufacturing with 3D-print technology. Rapid Prototyping $J 14$ : 167-172.

9. Nagata S (1994) Modification of the stages in total reconstruction of the auricle: Part IV Ear elevation for the constructed auricle. Plast Reconstr Surg 93: 254-266. [Crossref]

10. Kristiansen M, Öberg M, Wikström SO (2013) Patients" satisfaction after ear reconstruction with autologous rib cartilage. J Plast Surg Hand Surg 47: 113-117. [Crossref] 
11. Zhu P, Chen S (2016) Clinical outcomes following ear reconstruction with adjuvant 3D template model. Acta Oto-Laryngol 136: 1-6. [Crossref]

12. Baluch N, Nagata S, Park C, Wilkes GH, Reinisch J, et al. (2014) Auricular reconstruction for microtia: A review of available methods. Plast Surg-Chir Plas 22: 39-43. [Crossref]

13. Ross RJ, Shayan R, Mutimer KL, Ashton MW (2013) Autologous fat grafting: current state of the art and critical review. Ann Plas Surg 73: 352-357. [Crossref]

14. Bauer BS (2009) Reconstruction of microtia. Plast Reconstr Surg 124: S14e-S26e.
15. Nakai H (1990) Reconstruction of microtia. Pursuing a natural appearance. Clin Plast Surg 17: 287-304. [Crossref]

16. Maclennan A (2010) The artificial life route to artificial intelligence: Building embodied, situated agents. J Am Soc Inf Sci 47: 482-483.

17. Russell S, Dewey D, Tegmark M (2016) Research Priorities for Robust and Beneficia Artificial Intelligence. Japanese society for Artificial Intelligence: 32.

18. Russell SJ, Norvig P (2010) Artificial intelligence: a modern approach. Appl Mechan Mater 263: 2829-2833.

Copyright: $(02019$ Zhu P. This is an open-access article distributed under the terms of the Creative Commons Attribution License, which permits unrestricted use, distribution, and reproduction in any medium, provided the original author and source are credited. 